\title{
Chirp Multiplication by Four Wave Mixing for Wideband Swept-Frequency Sources for High Resolution Imaging
}

\author{
Naresh Satyan, Student Member, IEEE, George Rakuljic, and Amnon Yariv, Life Fellow, IEEE
}

\begin{abstract}
We present an analysis and demonstration of the doubling of the chirp rate and total chirp bandwidth of a frequency chirped optical signal by the process of four-wave mixing in a non-linear optical medium. The effects of chromatic dispersion and input power on the maximum achievable output bandwidth are analyzed, and a dispersion compensation technique for phase matching is described. The doubling of an input linear frequency sweep of $100 \mathrm{GHz} / 1 \mathrm{~ms}$ in a highly nonlinear optical fiber is experimentally demonstrated. Further, it is proposed that a cascaded implementation of the four-wave mixing process leads to a geometric increase in the bandwidth of the frequency chirp. With an electronically tuned chirped laser at the input stage, this process can be used to generate extremely wideband swept frequency sources with no moving parts, for applications in high-speed and high-resolution optical imaging and spectroscopy.
\end{abstract}

Index Terms-Frequency conversion, laser radar, nonlinear optics, optical imaging.

\section{INTRODUCTION}

$\mathbf{F}$ REQUENCY chirped optical waveforms with large frequency chirp range (optical bandwidth) have applications in high resolution optical imaging, LIDAR and infrared and Terahertz spectroscopy. The spatial resolution of an imaging system using a chirped laser source is inversely proportional to the chirp bandwidth [1], and the unambiguous range of the distance measurement is governed by the coherence length of the laser. Optical ranging applications therefore benefit from rapidly tunable, wide-bandwidth, and narrow linewidth swept-frequency optical sources. Rapidly swept laser sources with wide tuning ranges of $\sim 10-20 \mathrm{THz}$ also find applications in swept source Optical Coherence Tomography (OCT) [2]. Fourier-domain mode locking [3] and quasi-phase continuous tuning [4] have been developed to further improve the tuning speed and lasing properties of wideband swept-frequency sources, but these approaches suffer from complex mechanical embodiments that limit their linearity, coherence, reliability and

Manuscript received October 27, 2009; revised March 22, 2010; accepted May 25, 2010. Date of publication June 01, 2010; date of current version July 20, 2010. This work was supported in part by the Caltech Lee Center for Advanced Networking and in part by the Defense Advanced Research Projects Agencys Strategic Technology Office (Contract No. HR0011-10-C-0064).

N. Satyan is with the Department of Electrical Engineering, California Institute of Technology, Pasadena, CA 91125 USA (e-mail: naresh@ caltech.edu).

G. Rakuljic is with Telaris Inc., Santa Monica, CA 90403 USA (e-mail: rakuljic@telarisinc.com).

A. Yariv is with the Department of Applied Physics and the Department of Electrical Engineering, California Institute of Technology, Pasadena, CA 91125 USA (e-mail: ayariv@caltech.edu).

Digital Object Identifier 10.1109/JLT.2010.2051659 ease of use and manufacture. Single mode semiconductor lasers (SCLs) are attractive candidates for the generation of wideband chirped waveforms owing to their narrow linewidths and the ability to electronically control the lasing frequency via the injection current. We have previously demonstrated the generation of precisely controllable optical frequency sweeps using an SCL in an optoelectronic phase-lock loop [5]. However, the bandwidth over which the frequency of a single mode SCL can be tuned is typically limited to a few hundred $\mathrm{GHz}$, which is insufficient for high resolution LIDAR and OCT applications.

In this paper, we propose and demonstrate the doubling of the bandwidth of a chirped optical waveform by the process of Four-Wave Mixing (FWM) in a nonlinear optical medium. It is a well known observation [6] that the dithering of the pump signal to suppress Stimulated Brillouin Scattering (SBS) in a FWM experiment produces a broadening of the idler signal; this broadening is generally regarded as an undesirable side effect. We theoretically and experimentally demonstrate that the frequency chirp characteristics of the pump signal are faithfully reproduced in the idler, which implies that the chirp-doubled signal can be used for higher resolution optical imaging. The effect of chromatic dispersion on the maximum achievable output bandwidth is analyzed, and a dispersion compensation technique to reduce the required input power levels is described. We show that this approach can be cascaded to achieve a geometrical increase in the output chirp bandwidth and that the chirp bandwidth can be tripled using two chirped input fields. Finally, we present the design of a cyclical FWM "engine" to achieve large output chirp bandwidths using a single non-linear waveguide.

\section{THEORY}

\section{A. Bandwidth Doubling by Four Wave Mixing (FWM)}

Consider the experiment shown in Fig. 1. A chirped optical wave and a reference monochromatic wave are coupled together, amplified, and fed into a non-linear optical waveguide with a large third order non-linear susceptibility $\chi^{(3)}$ and a relatively low group velocity dispersion $(\mathrm{GVD})$ parameter $D_{c}$. Highly non-linear fibers (HNLF), photonic crystal fibers, higher order mode (HOM) optical fibers [7], semiconductor optical amplifiers (SOAs) [8] and integrated silicon waveguides [9] can be used to provide the necessary non-linear susceptibility and control over the GVD. In this paper, we will assume that the non-linear medium is a highly non-linear optical fiber. An optical filter, typically based on a diffraction grating, is used at the output to select the waveform of interest. 


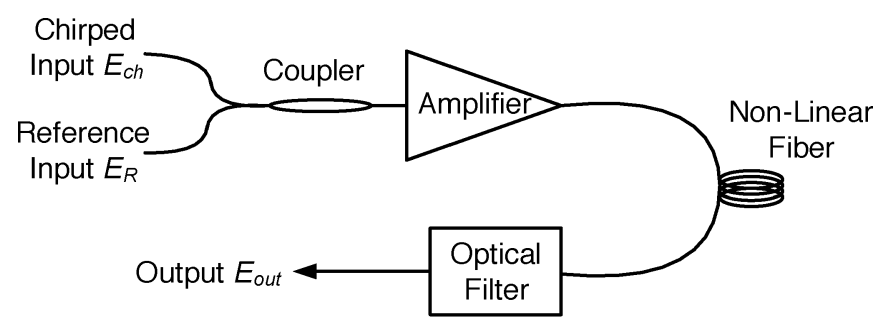

(a)

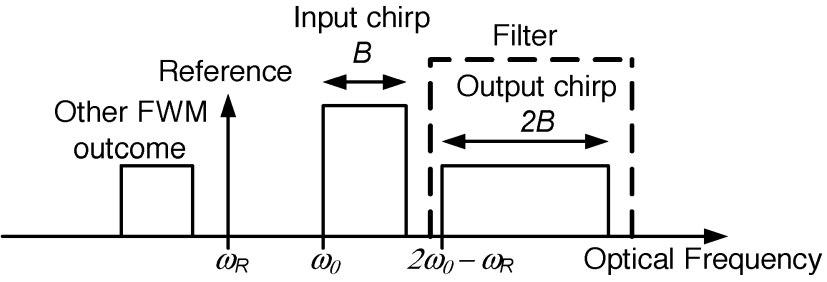

(b)

Fig. 1. (a) Schematic diagram of the Four-Wave Mixing (FWM) experiment for chirp bandwidth doubling. (b) Spectral components of the input and FWMgenerated fields. The chirp-doubled component is optically filtered to obtain the output waveform.

Let the electric fields of the chirped and the reference waves be given by

$$
\begin{aligned}
E_{\mathrm{ch}}(z, t) & =\frac{1}{2} A_{\mathrm{ch}}(z) \exp \left(j\left(\omega_{0} t+\phi(t)-\beta_{\mathrm{ch}} z\right)\right)+\text { c.c. } \\
E_{R}(z, t) & =\frac{1}{2} A_{R}(z) \exp \left(j\left(\omega_{R} t-\beta_{R} z\right)\right)+\text { c.c. }
\end{aligned}
$$

where $\phi(t)$ represents the optical chirp. The fields are assumed to be linearly polarized along the same axis, and $z$ is the direction of propagation. The propagation vectors $\beta$ are determined by the waveguide. The instantaneous frequency of the chirped wave is given by

$$
\omega_{\mathrm{ch}}(t)=\omega_{0}+\frac{d \phi}{d t}
$$

For the particular case of a linearly chirped wave, $\phi(t)=\xi t^{2} / 2$, and $\omega_{\mathrm{ch}}(t)=\omega_{0}+\xi t$. Typical optical frequency chirps of interest for imaging exceed bandwidths of $100 \mathrm{GHz}$ in a time less than $1 \mathrm{~ms}$, and SBS effects can be neglected in this analysis. The rate of the optical chirp is several orders of magnitude slower than the optical frequency, and the chirped wave can therefore be regarded as a monochromatic wave of frequency $\omega_{\mathrm{ch}}(t)$. The chirped and reference waves interact in the nonlinear fiber through the FWM process to give rise to a nonlinear polarization [10]

$$
P_{\mathrm{NL}}=4 \chi^{(3)}: E E E,
$$

where $E$ is the vector sum of the electric fields in (1). Among the various frequency terms which are present in the triple product (3) is the term

$$
P_{\mathrm{NL}}(z, t) \propto A_{\mathrm{ch}}^{2} A_{R}^{*} \exp \left(j\left(\left(2 \omega_{0}-\omega_{R}\right) t+2 \phi(t)\right)\right),
$$

which radiates a wave of frequency

$$
\omega_{\text {out }}(t)=2 \omega_{0}-\omega_{R}+2 \frac{d \phi}{d t}=2 \omega_{\mathrm{ch}}(t)-\omega_{R} .
$$

This process can be described quantum mechanically by the annihilation of two photons of the chirped field to create a photon of the reference field and a photon of the output field. Comparing (5) and (2), we see that the output chirp is twice the input chirp. By the proper selection of the input frequencies $\omega_{0}$ and $\omega_{R}$, the output waveform can be separated out by an optical filter, as shown in Fig. 1(b). If the bandwidth of the input chirp is $B$ $(\mathrm{rad} / \mathrm{s})$, the necessary condition for filtering the output waveform is

$$
\Delta \omega(t) \doteq \omega_{\mathrm{ch}}(t)-\omega_{R} \geq B
$$

Note that the output optical wavelength is in the same region as the input, and the output can therefore be amplified and re-used in a cascaded scheme as discussed in Section IV.

The expression for the output optical power can be obtained following a straightforward derivation [11] as outlined below. We restrict ourselves to the output electric field of the form

$$
E_{\text {out }}(z, t)=\frac{1}{2} A_{\text {out }}(z) \exp \left(j\left(\omega_{\text {out }} t-\beta_{\text {out }} z\right)\right)+\text { c.c. }
$$

which is generated by plugging the nonlinear polarization (3) into the nonlinear wave equation

$$
\frac{\partial^{2} E}{\partial z^{2}}=\frac{n^{2}}{c^{2}} \frac{\partial^{2} E}{\partial t^{2}}+\frac{\alpha n}{c} \frac{\partial E}{\partial t}+\mu_{0} \frac{\partial^{2} P_{\mathrm{NL}}}{\partial t^{2}},
$$

where $n$ is the refractive index in the fiber and $\alpha$ represents the loss per unit length. The input chirped and reference fields are assumed to be undepleted, i.e.,

$$
A_{\mathrm{ch}, R}(z)=A_{\mathrm{ch}, R}(0) \exp (-\alpha z / 2)
$$

and $A_{\text {out }}(z)$ is assumed to be slowly growing along the waveguide, i.e., $\partial^{2} A_{\text {out }} / \partial z^{2} \ll \beta_{\text {out }} \partial A_{\text {out }} / \partial z$. The differential equation for the output field is then given by

$$
\begin{aligned}
& \frac{d A_{\text {out }}}{d z}=-\frac{\alpha}{2} A_{\text {out }}-\frac{j n c \epsilon_{0} \gamma A_{\mathrm{eff}}}{2} A_{\mathrm{ch}}^{2}(0) A_{R}^{*}(0) \\
& \times e^{-3 \alpha z / 2} e^{-j \Delta \beta z}
\end{aligned}
$$

where $A_{\mathrm{eff}}$ is the effective area of the mode in the fiber, $\gamma$ is the nonlinear coefficient of the fiber, given by

$$
\gamma=\frac{3 \omega \mu_{0} \chi^{(3)}}{n^{2} \epsilon_{0} A_{\mathrm{eff}}}
$$

and $\Delta \beta$ is the phase mismatch defined as

$$
\Delta \beta \doteq 2 \beta_{\mathrm{ch}}-\beta_{R}-\beta_{\text {out }}
$$

The phase mismatch is a function of the frequency difference between the chirped wave and the reference wave. Ignoring the 


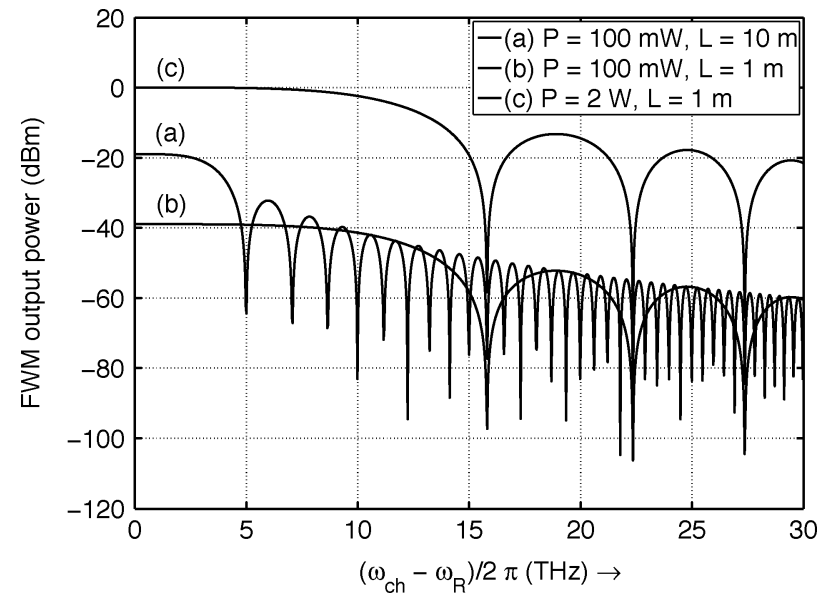

Fig. 2. Output power as a function of the input frequency difference, for different values of fiber length and input power $\left(P_{\mathrm{ch}}=P_{R}=P\right)$. The dispersion, loss and non-linear coefficient of the fiber are described in the text.

effect of self phase modulation of the chirped beam (which is valid when the input power is low), (12) can be written as

$$
\Delta \beta=-2 \sum_{k=1}^{\infty} \frac{\beta_{2 k}}{(2 k) !}(\Delta \omega)^{2 k},
$$

where $\Delta \omega$ is defined in (6) and $\beta_{k}$ is the $k$ th derivative of $\beta(\omega)$, evaluated at $\omega=\omega_{\mathrm{ch}} \cdot \beta_{2}$ is related to the GVD parameter $D_{c}$ by $\beta_{2}=-\lambda^{2} D_{c} /(2 \pi c)$.

\section{B. Bandwidth Limitations Due to Dispersion}

Integrating (10), we derive the output power after propagation through a distance $L[11]$ :

$$
\begin{aligned}
P_{\text {out }}(L)=\gamma^{2} P_{\mathrm{ch}}^{2} P_{R} e^{-\alpha L} & \left(\frac{1-e^{-\alpha L}}{\alpha}\right)^{2} \frac{\alpha^{2}}{\alpha^{2}+\Delta \beta^{2}} \\
& \times\left(1+\frac{4 e^{-\alpha L} \sin ^{2} \frac{\Delta \beta L}{2}}{\left(1-e^{-\alpha L}\right)^{2}}\right) .
\end{aligned}
$$

From (13) and (14), the maximum value of the input frequency separation, and hence the output chirp bandwidth, will ultimately be limited by the phase mismatch in the fiber. Consider as an example, a commercially available dispersion flattened HNLF with a non-linear coefficient $\gamma=11.3 \mathrm{~km}^{-1} \mathrm{~W}^{-1}$, loss $\alpha=1 \mathrm{~dB} / \mathrm{km}$, and dispersion parameter $D_{c}=0.5 \mathrm{ps} / \mathrm{nm} \cdot \mathrm{km}$. For this dispersion-flattened fiber, higher order dispersion terms ( $\beta_{4}$ and above in (13)) can be neglected. Let us assume that the chirp and reference powers are equal, i.e., $P_{\mathrm{ch}}=P_{R}$. The output power as a function of the input frequency separation $\left(\omega_{\mathrm{ch}}-\omega_{R}\right)$, for various values of input power $P_{\mathrm{ch}}$ and fiber length $L$, is calculated using (13) and (14) and plotted in Fig. 2. The FWM bandwidth $B_{\mathrm{FWM}}$ can be defined as the maximum input frequency separation over which useful output power is generated, which is here taken to be the $-3 \mathrm{~dB}$ point. It is important to note that the filtering condition (6) implies that $B_{\mathrm{FWM}}$ is equal to the maximum possible output bandwidth. The maximum fiber lengths and the input power requirements for different values of output bandwidth and output power are summarized in Table I.
TABLE I

LENGTH OF HNLF AND INPUT POWER REQUIREMENTS FOR DIFFERENT OUTPUT BANDWIDTHS AND POWER LEVELS

\begin{tabular}{llll}
\hline \multirow{2}{*}{$\begin{array}{l}\text { Output } \\
\text { bandwidth } \\
(\mathrm{THz})\end{array}$} & $\begin{array}{l}\text { Maximum } \\
\text { fiber } \\
\text { length }(\mathrm{m})\end{array}$ & \begin{tabular}{l} 
Input power required \\
\cline { 3 - 4 }
\end{tabular} & $P_{\text {ch }}=P_{R}(\mathrm{dBm})$ \\
\hline 1 & 105 & 19.5 & 16.2 \\
5 & 4.3 & 29.0 & 25.4 \\
10 & 1.1 & 32.8 & 29.5 \\
15 & 0.45 & 35.2 & 32.0 \\
\hline
\end{tabular}

It is clear from Fig. 2 and Table I that the maximum output bandwidth is determined by the length of fiber used in the experiment. For a given value of the dispersion parameter, $B_{\mathrm{FWM}}$ reduces as $L$ is increased. To obtain larger bandwidths, a fiber with lower dispersion must be used. For a given length of fiber, the output power level depends only on the input power. For example, for a desired output bandwidth of $10 \mathrm{THz}$ and an output power of $0 \mathrm{dBm}$, the maximum (dispersion-limited) fiber length is $1.1 \mathrm{~m}$, and the input power required is $P_{\mathrm{ch}}=P_{R}=1.9 \mathrm{~W}$. This power level can be achieved with high power fiber amplifiers, but is desirable that commercially available telecom-grade erbium doped fiber amplifiers with output powers of $\sim+20$ $\mathrm{dBm}$ be used to reduce the system cost. In the following section, we describe a quasi-phase matching technique using dispersion compensation to achieve this target.

It was assumed in the preceding discussion that higher order terms in the dispersion curve $\beta(\omega)$ were small and could be neglected. This description works well for dispersion-flattened HNLF, but other nonlinear fibers can have a significant contribution to the phase mismatch arising from the next higher order dispersion, i.e., $\beta_{4}$. The output bandwidth limit can for this case be calculated exactly using (13) in (14). A rough estimate for the bandwidth limit can be obtained by comparing the contributions to the phase mismatch due to $\beta_{2}$ and $\beta_{4}$. Using (13), these contributions become equal at $\Delta \omega_{1} \sim \sqrt{\left|12 \beta_{2} / \beta_{4}\right|}$. If $\Delta \omega_{1}$ is less than $B_{\mathrm{FWM}}$ calculated using only the phase mismatch due to $\beta_{2}$, then the maximum achievable output bandwidth is approximately given by $\Delta \omega_{1}$.

\section{Quasi-Phase Matching Using Alternating Dispersion Stages}

It is desirable to increase the length of the non-linear fiber used in the experiment, so as to increase the interaction length for the FWM process, thereby reducing input power requirements. However, the length cannot be increased arbitrarily, since the phase mismatch causes a reduction in the overall output power. This limitation can be overcome by using a multi-segment HNLF where the sign of the dispersion parameter of a segment is alternatively chosen to be positive or negative, as shown in Fig. 3(a). The dispersion parameter $D_{c}$ is changed by engineering the waveguide dispersion differently in the alternating segments. We again make the assumption of a dispersion-flattened fiber where $\beta_{4}$ can be neglected. Dispersion-flattened HNLFs with dispersion parameters in the range of -1.0 to $+1.5 \mathrm{ps} / \mathrm{nm} \cdot \mathrm{km}$ at $1550 \mathrm{~nm}$ are readily available. An exact expression for the output field is easily obtained by integrating 


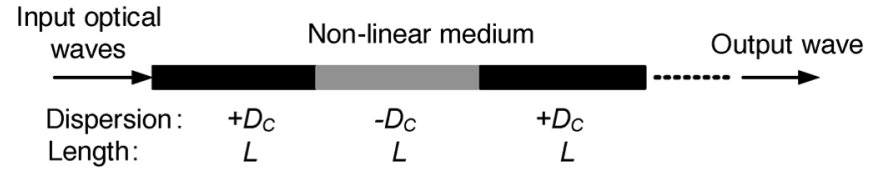

(a)
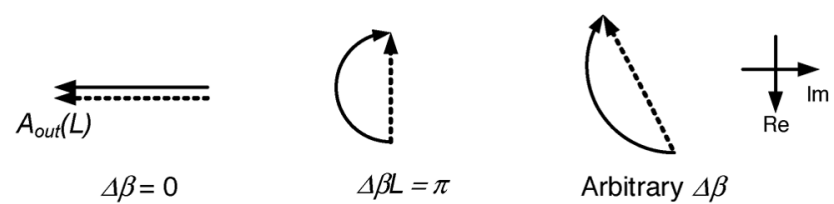

(b)

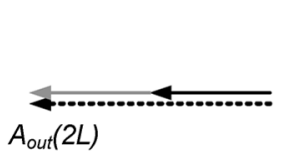

$\Delta \beta=0$

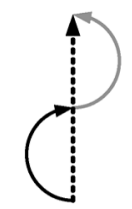

$\Delta \beta L=\pi$

(c)

Fig. 3. (a) Multi-segment alternating dispersion waveguide for quasi-phase matching. The evolutions of the output field $A_{\text {out }}(z)$ along the waveguide for one and two segments are shown in (b) and (c) respectively. The dashed lines represent the field at (b) $z=L$ and (c) $z=2 L$.

(10) over the entire structure, but we present below an intuitive explanation of the power build-up in the fiber. For a low loss fiber, we can set $\alpha=0$ in (10) to obtain the simple differential equation

$$
\frac{d A_{\mathrm{out}}}{d z}=-\frac{j n c \epsilon_{0} \gamma A_{\mathrm{eff}}}{2} A_{\mathrm{ch}}^{2}(0) A_{R}^{*}(0) e^{-j \Delta \beta z} .
$$

The solution to this equation is a phasor that traces out a circle in the complex plane as the distance $z$ is increased, as shown in Fig. 3(b). The maximum value of the field occurs when $z_{\max } \Delta \beta=\pi$. As $z$ is increased beyond this value, the magnitude of the field phasor decreases, and the power output decreases. When the sign of the dispersion parameter is reversed, the sign of $\Delta \beta$ is also reversed according to (13), and the field phasor now traces out a circle of the opposite sense, as depicted in Fig. 3(c). By symmetry considerations, the total output field at the end of the second segment is equal to twice the value of the field at the end of the first segment, for any arbitrary value of $\Delta \beta$. For a structure with $N$ alternating segments, the output field scales as $N$, and the output power scales as $N^{2}$. The variation of $P_{\text {out }}$ along a structure with three alternating segments of HNLF, calculated using (10), is plotted in Fig. 4, clearly showing the quadratic scaling of the output power with number of segments. Conversely, for a given desired output power, the input power requirement is reduced. For the HNLF example considered in Section II.B, an output bandwidth of $10 \mathrm{THz}$ and output power of $0 \mathrm{dBm}$ can be achieved using a structure with 30 segments of length $\mathrm{L}=1.1 \mathrm{~m}$ and alternating dispersions of $\pm 0.5 \mathrm{ps} / \mathrm{nm} \cdot \mathrm{km}$, with an input power of only $200 \mathrm{~mW}$, as opposed to an input power requirement of $1.9 \mathrm{~W}$ if a single segment were used.

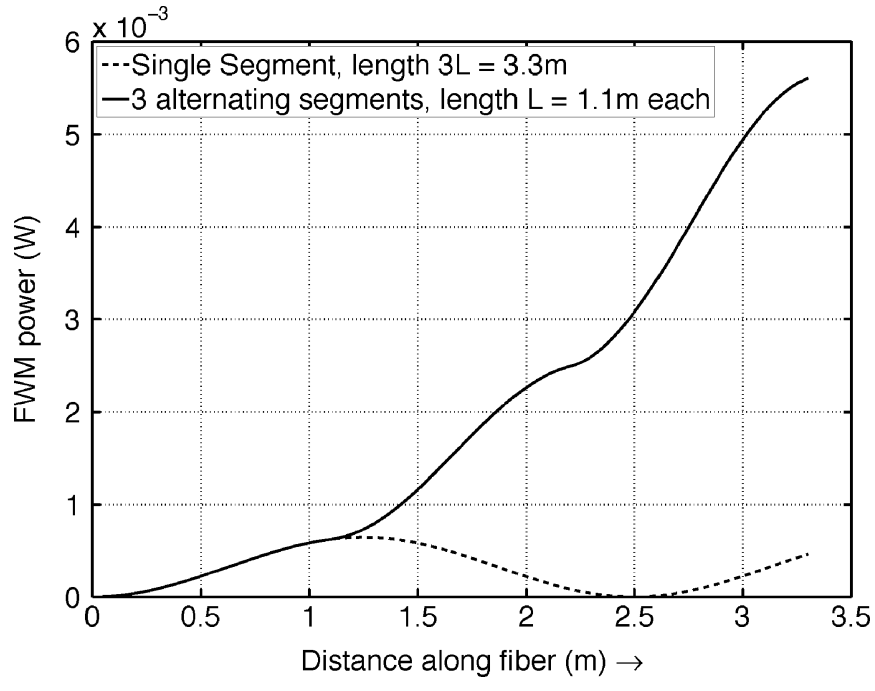

Fig. 4. Comparison of the generated FWM field in a structure with 3 segments of lengths $L$ each and alternating dispersions of $\pm D_{c}$, with a single segment of length $3 L$ and dispersion $+D_{c}$. The values used in the calculations were $L=1.1 \mathrm{~m}$ and $D_{c}=0.5 \mathrm{ps} / \mathrm{nm} \cdot \mathrm{km}$.

The number of segments that can be used in this technique is limited by the insertion loss due to the fiber splices. Let the ratio of the transmitted to the incident field amplitudes at a fiber splice be given by $t$, and let $F^{(k)}$ denote the amplitude of the FWM field generated in the $k$ th segment. The fields generated in all the segments add in phase. The chirped and reference fields in the $k$ th segment are given by $A_{\mathrm{ch}, R}^{(k)}=t^{k-1} A_{\mathrm{ch}, R}^{(1)}$, and the FWM field generated in the $k$ th segment is consequently given by $F^{(k)}=t^{3 k-3} F^{(1)}$. The output field after the $k$ th stage is therefore given by

$$
A_{\mathrm{out}}^{(k)}=t A_{\mathrm{out}}^{(k-1)}+t^{3 k-3} F^{(1)},
$$

which can be solved to yield

$$
P_{\mathrm{out}}^{(k)}=\left(\frac{t^{k-1}\left(1-t^{2 k}\right)}{1-t^{2}}\right)^{2} P_{\mathrm{out}}^{(1)}
$$

Under the assumption that $(1-t) \ll 1$, (17) reduces to

$$
\frac{P_{\text {out }}^{(k)}}{P_{\text {out }}^{(1)}} \approx k^{2} t^{2(k-1)}
$$

It is therefore crucial to minimize the splice losses in order to increase the FWM interaction length. In the absence of splice losses, the number of segments is limited by material loss in the waveguide, and the total achievable bandwidth is ultimately limited by the gain bandwidth of the amplifiers used in the experiment.

It should be noted that quasi-phase matched FWM using a similar concept has been demonstrated theoretically and experimentally [12], [13], where the phase mismatch accumulated during the FWM process is periodically compensated for using a dispersion compensating fiber (DCF) or a single mode fiber (SMF). In the process described in this section, the quasi-phase 


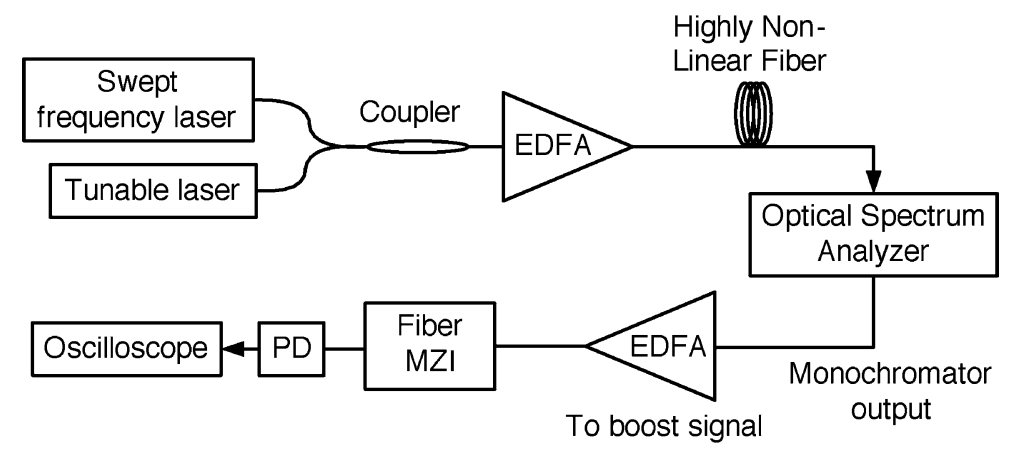

Fig. 5. Schematic diagram of the experimental setup for the demonstration of chirp bandwidth doubling by Four Wave Mixing. EDFA: Erbium doped fiber amplifier, MZI: Mach-Zehnder Interferometer, PD: Photodetector. The differential delay in the MZI is approximately $2.7 \mathrm{~ns}$.

matching is achieved using nonlinear fiber. This is an important distinction since the use of SMF or DCF will require two fiber splices per segment of HNLF, which then leads to a lower achievable gain from (18). Further, the loss per splice is also expected to be higher, since dissimilar fibers have to be spliced together.

We have again neglected the effect of higher order dispersion terms in the preceding analysis. In the presence of nonnegligible higher order dispersion terms, perfect quasi-phase matching can only be achieved by reversing the signs of all the terms $\beta_{2 k}$ in (13), for $k=1,2, \ldots$ However, a degree of quasi-phase-matching can still be achieved by reversing the sign of the dispersion parameter $D_{c}$. The modification to the output power due to the effect of higher order terms can be determined exactly by integrating (10).

\section{EXPERIMENT}

A schematic diagram of the proof-of-principle experimental setup is shown in Fig. 5. The input chirped wave was a transform limited, linearly chirped waveform that sweeps $100 \mathrm{GHz}$ in $1 \mathrm{~ms}$, generated by a semiconductor DFB laser in an optoelectronic phase-lock loop [5]. The linearity of the chirp is very important for imaging and optical ranging applications, since it eliminates the need for extensive post-processing. A tunable laser (Agilent Technologies) was used as the monochromatic reference wave. The two optical waves were coupled using a polarization maintaining coupler, amplified using an EDFA and fed into a commercial dispersion flattened HNLF. The HNLF had a gain $\gamma=11.3 \mathrm{~km}^{-1} \mathrm{~W}^{-1}$, loss $\alpha=1 \mathrm{~dB} / \mathrm{km}$, length $L=$ $100 \mathrm{~m}$, and dispersion parameter $D_{c}=+1.2 \mathrm{ps} / \mathrm{nm} \cdot \mathrm{km}$. The output of the HNLF was measured on an optical spectrum analyzer, and is shown in Fig. 6. The figure clearly shows the generation of a frequency doubled FWM output that sweeps over an optical bandwidth of $200 \mathrm{GHz}$. A second FWM component sweeping over $100 \mathrm{GHz}$ in the reverse direction was generated on the low frequency side, corresponding to the FWM process involving two photons of the reference wave and one photon of the chirped wave. The experimentally measured values of the output fields are in excellent agreement with the theoretical calculation based on the measured input powers and (13) and (14).

The ability of the experiment to reproduce the dynamic characteristics of the input optical frequency chirp at the output was also verified. The output waveform was filtered out using the

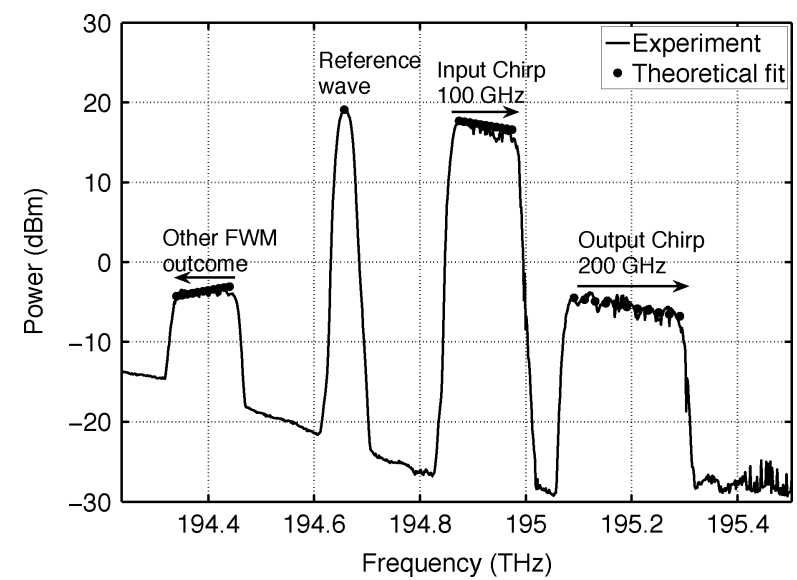

Fig. 6. Experimental demonstration of bandwidth doubling by Four Wave Mixing. The reference wave was monochromatic (resolution limited) and the input chirp bandwidth was $100 \mathrm{GHz}$. The arrows indicate the direction of the chirp. The second FWM product, generated at the lower frequency, chirps in the opposite direction. The theoretical FWM power was calculated using (13) and (14) using the measured input powers.

monochromator output of the optical spectrum analyzer, and amplified using a telecom EDFA. The input and output frequency chirps were characterized by passing them through a Mach-Zehnder interferometer (MZI) with time delay $\tau_{\mathrm{MZI}}=$ $2.7 \mathrm{~ns}$, as shown in Fig. 5. The frequency of the detected photocurrent is related to the slope $\xi$ of the optical chirp by $\omega=$ $\xi \tau_{\mathrm{MZI}}$. The spectrograms of the photocurrents are calculated and plotted in Figs. 7(a) and (b). The results clearly show that the optical chirp rate is doubled by the FWM process from $10^{14}$ $\mathrm{Hz} / \mathrm{s}$ to $2 \times 10^{14} \mathrm{~Hz} / \mathrm{s}$, and the transform-limited linearity of the input chirp is maintained at the output, making the output frequency chirped waveform suitable for 3-D imaging applications. The FWM technique can also be used to increase the chirp rate of swept frequency optical waveforms.

\section{BANDWIDTH EXTENSION}

The FWM process demonstrated in this paper generates a chirp-doubled optical wave in the same wavelength range as the input signal. The frequency spacing between the output chirp and the input chirp is only limited by the sharpness of the optical filter used to filter out the output. Using diffraction grating based filters, this gap can be as small as a few GHz. It has been demonstrated by Ishida and Shibata [14] that the FWM process can 


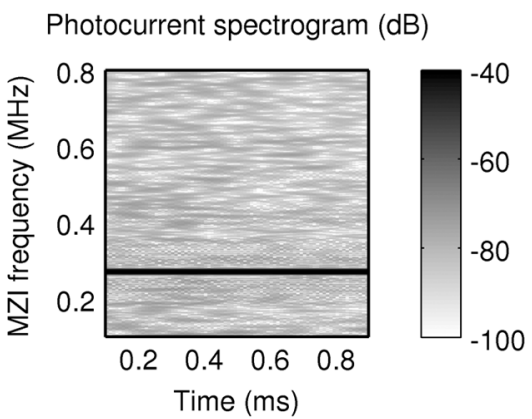

(a)

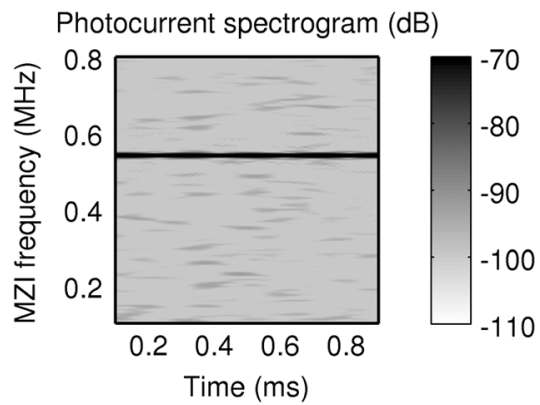

(b)

Fig. 7. Spectrograms of the photocurrent detected at the output of the Mach-Zehnder interferometer shown in Fig. 5, for the (a) input and (b) output optical chirps. The frequency of the detected photocurrent is proportional to the slope of the optical frequency chirp.

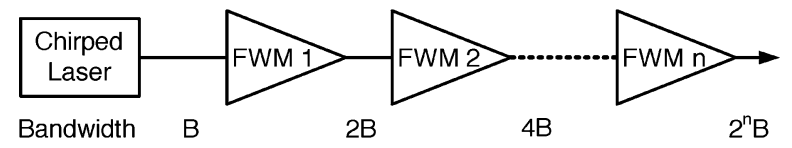

Fig. 8. Cascaded Four Wave Mixing (FWM) stages for geometric scaling of the chirp bandwidth. Each stage consists of a coupler, amplifier, HNLF and filter as shown in Fig. 1(a).

be cascaded to geometrically increase the frequency separation between the two input signals. This principle can be extended to chirped signals to achieve geometric increases in the chirp bandwidth. The output chirped signal from the FWM experiment can be filtered, amplified again using an EDFA and mixed with the same reference signal in an HNLF to further double the chirp bandwidth. A cascade of $n$ such stages leads to the geometric scaling of the output bandwidth by a factor $2^{n}$, as shown in Fig. 8. For example, starting with a $200 \mathrm{GHz}$ chirped semiconductor laser at the input, an output bandwidth of $12.8 \mathrm{THz}$ is obtained after $n=6$ stages. Note that the same reference monochromatic signal can be used for each stage, since the filtering condition (6) is always satisfied if it is satisfied for the first FWM stage. If the dispersion compensation technique for quasi-phase matching described in Section II.C is used, the total output bandwidth is only limited by the gain bandwidth of the amplifiers used in the experiment, and by additional noise introduced by the amplification stages.

The FWM process fundamentally involves the interaction of three input fields to produce the output field. An optimum use of the process for bandwidth multiplication can therefore result in bandwidth tripling, and not just doubling, as described below.

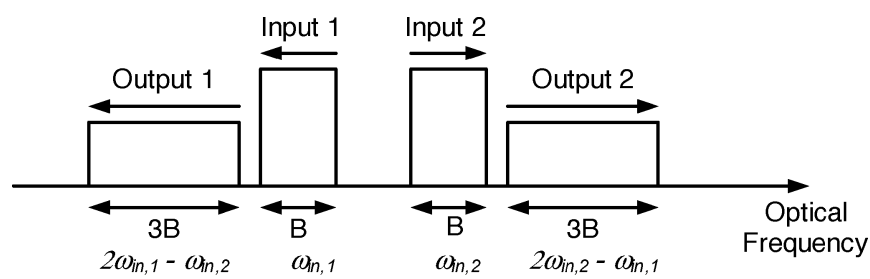

Fig. 9. Spectral components in a bandwidth tripling FWM experiment using two chirped optical inputs.

Let the monochromatic reference wave of Fig. 1 be replaced by a chirped wave that sweeps in the direction opposite to the original chirp. We now have two input chirped waves which are mirror images of each other, with frequencies given by

$$
\begin{aligned}
& \omega_{\text {in }, 1}=\omega_{0}-B_{0}-\frac{d \phi}{d t} \\
& \omega_{\text {in }, 2}=\omega_{0}+B_{0}+\frac{d \phi}{d t},
\end{aligned}
$$

where $\omega_{0}$ and $B_{0}$ are constants. The two output fields generated by two distinct FWM processes have frequencies

$$
\begin{aligned}
& \omega_{\text {out }, 1}=2 \omega_{\text {in }, 1}-\omega_{\text {in }, 2}=\omega_{0}-3 B_{0}-3 \frac{d \phi}{d t} \\
& \omega_{\text {out }, 2}=2 \omega_{\text {in }, 2}-\omega_{\text {in }, 1}=\omega_{0}+3 B_{0}+3 \frac{d \phi}{d t} .
\end{aligned}
$$

The output waveforms have bandwidths that are thrice the bandwidth of the individual input chirps, as shown in Fig. 9. Further, the two output waveforms can be amplified and used in a cascaded process similar to the one described for the bandwidth doubling approach, to achieve a geometrical bandwidth scaling of $3^{n}$. Starting with two frequency sweeps of $200 \mathrm{GHz}$ each, a chirp bandwidth of $16.2 \mathrm{THz}$ can now be achieved using $n=4$ stages.

The geometric enhancement of the chirp bandwidth using a cascade of $n$ stages has the drawback that it requires $n$ amplifiers and $n$ non-linear waveguides, thereby increasing the overall system cost. This can be overcome by folding back the cascaded process using a FWM "engine" as shown in Fig. 10(a). The input chirped wave sweeps over a bandwidth $B$ during a time $T$, and is then turned off. A monochromatic reference wave is also coupled into the nonlinear medium. The FWM output of bandwidth $2 B$ is selected by the optical filter, delayed by a time $T$, amplified and fed back into the non-linear fiber as the chirped input. From time $T$ to $2 T$, the optical filter is configured to select the new FWM output of bandwidth $4 B$. The combination of optical filter configuration and the delay $T$ therefore ensures that only two optical waves are input into the non-linear fiber at a given instant of time. The slope of the frequency chirp at the output port then increases geometrically with time, as depicted in Fig. 10(b). The amount of practically achievable delay $T$ imposes a lower bound on the input optical chirp rate, for a given chirp bandwidth. A fiber delay of $20 \mathrm{~km}$ provides a delay of $100 \mu \mathrm{s}$, which is quite sufficient for sweeping typical semiconductor lasers, and switching the optical filters. This approach can be easily modified to include two chirped inputs. 


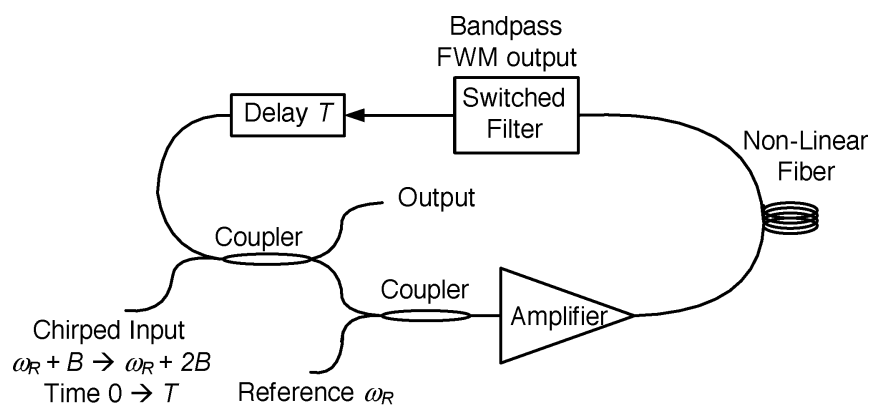

(a)

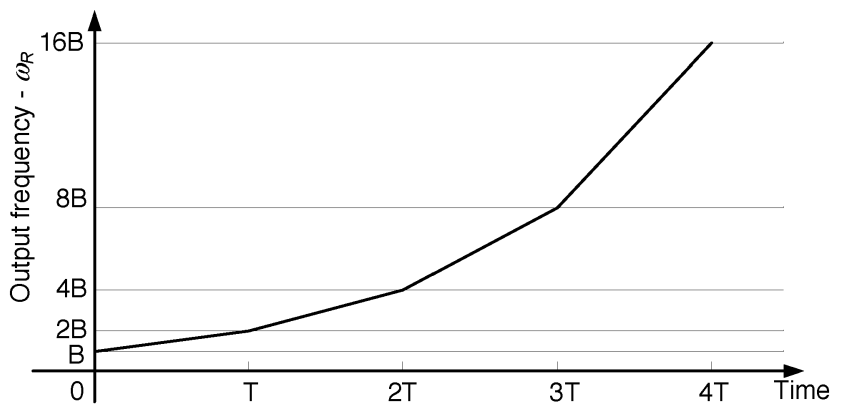

(b)

Fig. 10. (a) FWM "engine" for geometric scaling of the chirp bandwidth. The filter is switched every $T$ seconds so that it passes only the FWM component generated. (b) Output frequency versus time.

\section{ACKNOWLEDGMENT}

The authors thank Profs. K. Vahala and A. Scherer at Caltech for the loan of EDFAs for use in the experiment.

\section{REFERENCES}

[1] W. S. Burdic, Radar Signal Analysis. Englewood Cliffs, NJ: Prentice-Hall, Inc, 1968.

[2] S. H. Yun, G. J. Tearney, J. F. de Boer, N. Iftimia, and B. E. Bouma, "High-speed optical frequency-domain imaging," Opt. Exp., vol. 11, no. 22, pp. 2953-2963, 2003.

[3] R. Huber, M. Wojtkowski, and J. G. Fujimoto, "Fourier domain mode locking (FDML): A new laser operating regime and applications for optical coherence tomography," Opt. Exp., vol. 14, no. 8, pp. 3225-3237, 2006.

[4] C. Chong, T. Suzuki, A. Morosawa, and T. Sakai, "Spectral narrowing effect by quasi-phase continuous tuning in high-speed wavelength-swept light source," Opt. Exp., vol. 16, no. 25, pp. 21 105-21 118, 2008.

[5] N. Satyan, A. Vasilyev, G. Rakuljic, V. Leyva, and A. Yariv, "Precise control of broadband frequency chirps using optoelectronic feedback," Opt. Exp., vol. 17, no. 18, pp. 15 991-15 999, 2009.

[6] F. S. Yang, M. E. Marhic, and L. G. Kazovsky, "CW fibre optical parametric amplifier with net gain and wavelength conversion efficiency > 1," Electron. Lett., vol. 32, no. 25, pp. 2336-2338, Dec. 1996.

[7] S. Ramachandran, S. Ghalmi, J. W. Nicholson, M. F. Yan, P. Wisk, E. Monberg, and F. V. Dimarcello, "Anomalous dispersion in a solid, silica-based fiber,” Opt. Lett., vol. 31, no. 17, pp. 2532-2534, 2006.
[8] S. Diez, C. Schmidt, R. Ludwig, H. G. Weber, K. Obermann, S. Kindt, I. Koltchanov, and K. Petermann, "Four-wave mixing in semiconductor optical amplifiers for frequency conversion and fast optical switching," IEEE J. Sel. Top. Quantum Electron., vol. 3, no. 5, pp. 1131-1145, Oct. 1997.

[9] W. Mathlouthi, H. Rong, and M. Paniccia, "Characterization of efficient wavelength conversion by four-wave mixing in sub-micron silicon waveguides," Opt. Exp., vol. 16, no. 21, pp. 16 735-16 745, 2008.

[10] A. Yariv, Quantum Electronics, 3rd ed. New York: Wiley, 1989.

[11] K. O. Hill, D. C. Johnson, B. S. Kawasaki, and R. I. MacDonald, "CW three-wave mixing in single-mode optical fibers," J. Appl. Phys., vol. 49, no. 10, pp. 5098-5106, 1978.

[12] M. E. Marhic and F. S. Yang, "High-nonlinearity fiber optical parametric amplifier with periodic dispersion compensation," J. Lightw. Technol., vol. 17, no. 2, pp. 210-215, Feb. 1999.

[13] J. Kim, O. Boyraz, J. H. Lim, and M. N. Islam, "Gain enhancement in cascaded fiber parametric amplifier with quasi-phase matching: Theory and experiment," J. Lightw. Technol., vol. 19, no. 2, pp. 247-251, Feb. 2001.

[14] O. Ishida and N. Shibata, "Laser frequency synthesis employing fiber four-wave mixing," IEEE Photon. Technol. Lett., vol. 4, no. 10, pp. 1171-1174, Oct. 1992.

Naresh Satyan (S'09) received the B.Tech. degree in electrical engineering from the Indian Institute of Technology Madras, Chennai, India and the M.S. degree in electrical engineering from the California Institute of Technology (Caltech), Pasadena, in 2005 and 2007 respectively. He is currently working toward the Ph.D. degree in electrical engineering at Caltech.

His research interests include semiconductor lasers, optical phase-lock loops, optical imaging and sensing, nonlinear optics, RF photonics and optoelectronics.

George Rakuljic received the M.S. and Ph.D. degrees in electrical engineering from the California Institute of Technology in 1983 and 1987, respectively. He is a founder of Telaris Inc. and currently serves as its Chief Executive Officer. Prior to Telaris, Dr. Rakuljic founded and served as Chief Technology Officer of Arroyo Optics, Inc. Previously, he was Vice President of R\&D at Accuwave Corporation and a Member of the Research Staff at the Northrop Research and Technology Center. He has numerous publications and patents in the fields of optics, materials science and communications, and has been responsible for the development of several optoelectronic products for the telecommunications industry.

Amnon Yariv (S'56-M'59-F'70-LF'95) received the B.S., M.S., and Ph.D. degrees in electrical engineering from the University of California, Berkeley, in 1954, 1956, and 1958, respectively.

In 1959, he joined Bell Telephone Laboratories, Murray Hill, NJ. In 1964, he joined the California Institute of Technology (Caltech), Pasadena, as an Associate Professor of electrical engineering, becoming a Professor in 1966. In 1980, he became the Thomas G. Myers Professor of electrical engineering and applied physics. In 1996, he became the Martin and Eileen Summerfield Professor of applied physics and Professor of electrical engineering. He took part (with various co-workers) in the discovery of a number of early solid-state laser systems, in the original formulation of the theory of nonlinear quantum optics, in proposing and explaining mode-locked ultrashort-pulse lasers, GaAs optoelectronics, in proposing and demonstrating semiconductor-based integrated optics technology, in pioneering the field of phase conjugate optics, and in proposing and demonstrating the semiconductor distributed feedback laser. He has published widely in the laser and optics fields and has written a number of basic texts in quantum electronics, optics, and quantum mechanics.

Dr. Yariv is a member of the American Academy of Arts and Sciences, the National Academy of Engineering, and the National Academy of Sciences. 